\title{
Foreign Body Ingestion in Children: A Hospital Based Experience in Riyadh
}

\author{
Zubair Khurshid*, Abdelbasit Elsayed Ali, Saad Abdul Aziz Al Hamidi And Turki Ibrahim Alnafisah \\ Department of Pediatric Surgery, King Saud Medical City, Riyadh
}

*Corresponding Author: Zubair Khurshid, Department of Pediatric Surgery, King Saud Medical City, Riyadh.

Received: July 12, 2019; Published: September 10, 2019

DOI: 10.31080/ASPE.2019.02.0141

\begin{abstract}
The ingestion of foreign bodies is a relatively common problem in children especially infants and toddlers. A variety of objects such as coins, batteries, magnets, nails, pins, needles etc. can be ingested and the complications can be grave. With the advent of gadgets and electronic toys, the number of magnets and battery ingestions have shown an increasing trend, and in some cases turned out to be lethal for children.

Aim: The primary aim is to estimate the magnitude of the problem at our hospital. The secondary objectives were to analyze the data for age, gender, nature of ingested foreign bodies, management and the outcome.

Materials and methods: The records of all the children who were admitted in KSMC Riyadh with foreign body ingestion from 01/01/2016 to 31/12/2016 were retrospectively reviewed. The data were analyzed and recorded for age, sex, clinical presentation, nature of foreign bodies, location on radiograph, interventions done and hospital stay.

Results: A total of 61 patients with history of foreign body ingestion were included in the study. The mean age was 5.13 years (median 4.9 years) with male to female ratio being 2.1: 1. Button batteries were the most common ingested foreign bodies in $39 \%$ of the patients and esophagus was the most common site for location of ingested foreign bodies in 39\% cases. Endoscopy was done in $62.5 \%$ of the patients and one patient underwent laparotomy. Seven (12\%) patients had complications with jejunal perforation in one patient and esophageal/gastric ulcers in 6 patients. Re endoscopy was done in 8\% of patients. No patient required any further interventions on follow up.

Conclusion: Foreign body ingestion is an increasing health hazard in children of the region with a high incidence of potentially lethal battery ingestion putting an additional burden on health care system.
\end{abstract}

Keywords: Riyadh; Ingestion; Endoscopy

Ingestion of foreign bodies is a relatively common problem in pediatric emergency setting. Children tend to explore the environment by inserting objects in their mouth; some of these items can be inevitably swallowed. The problem is especially encountered in infants and toddlers [1-3].

The American Association of Poison Control Centers in 2000 reported that more than $75 \%$ of foreign body ingestions occur in children aged less than 5 years [4]. More than $98 \%$ of these cases are accidental, involving ingestion of common household items such as batteries, coins, jewelry, safety pins and magnets. Fortunately more than $80 \%$ of these foreign bodies are known to pass spontaneously $[2,5]$.

Endoscopic removal is mandated in $10-20 \%$ of the cases with a very small percentage $(<1 \%)$ requiring a surgical intervention
$[1,6,7]$. However the rate of endoscopic intervention is much higher, ranges from 63 to $76 \%$, in patients with intentional ingestion with surgical intervention needed in 12 to 16 percent of patients [8,9].

Ingestion of multiple foreign bodies are common in children with developmental delay $[10,11]$ The medical attention in most cases is sought once the parents witness or report a foreign body ingestion and in such cases the patients are mostly asymptomatic $[1,6,12,13]$ and when symptoms occur they are mostly related to the location of the foreign body.

Foreign bodies in the esophagus can produce various symptoms including dysphagia, chest pain, stridor, drooling and occasionally airway obstruction. Gastrointestinal foreign bodies produce less specific symptoms, including abdominal pain, melena, and hematochezia. 
Most complications are caused by impaction of foreign bodies in the esophagus, especially in the case of anatomical defects or preexisting diseases [14-16]. Esophageal perforation may result in neck swelling, crepitus and pneumomediastinum. Adjacent vascular erosion and fatal bleeding was reported in literature. If perforation occurs in the stomach or intestines, fever, abdominal pain and tenderness may develop. Bowel obstruction by a foreign body may cause abdominal distension, pain, and tenderness.

Common sites for obstruction by an ingested foreign body include the cricopharyngeal area, middle one third of the esophagus, lower esophageal sphincter, pylorus and ileocecal valve [17-19].

A growing incidence of children with foreign body ingestion, presenting to our centre, has been observed over the past few years. Therefore, the study has been undertaken.

\section{Materials and Methods}

This retrospective, descriptive analytical hospital based study for the incidence of foreign bodies in children was conducted in the Department of Pediatric surgery KSMC Riyadh.

The hospital records of all the patients aged 0-14 years with a history of foreign body ingestion who were admitted in the hospital in one year $(1 / 1 / 2016$ to $31 / 12 / 2016)$ were retrospectively reviewed and data collected and analyzed. The data were collected based on questionnaire by the principal investigator and one of the co investigators.

\section{Exclusion criteria}

Passage of the foreign body prior to admission and/or aspiration or inhalation of the foreign body.

The study was conducted as per the underlying format and all details were recorded from the review of the patients' records in a formulated questionnaire.

A detailed relevant clinical history and demographic profile was recorded and findings of examination were noted. The findings of Biplane radiographs (anteroposterior and lateral) of neck, chest and abdomen recorded. The hospital course of those patients who were managed conservatively and their follow up radiology till the ingested foreign body passed out spontaneously was studied.

The patients who underwent interventions, the nature of intervention, timing of intervention after ingestion of foreign body and its timing after the arrival to ER, success rate, complications or need for second intervention were recorded.
The average hospital stay and complications -if any- were studied and analyzed. Post discharge follow up was limited to patients who presented to hospital for any need for secondary interventions and late complications.

\section{Statistical methods}

Descriptive statistical analysis has been carried out in the present study. Results on continuous measurements are presented on Mean \pm SD and results on categorical measurements are presented in number percent (\%).

\section{Statistical software}

The Statistical software namely SPSS 15.0, MedCalc 9.0.1 and GraphPad Prism 6 was used for the analysis of the data and for graphs and figures.

\section{Results}

A total number of 61 patients were identified and data were collected and analyzed.

The minimum age was 1 month and maximum age was 13.9 years. Mean age is $5.13 \pm 2.68$ years with a median age of 4.9 years. Males formed the majority of the patients (41 males vs 20 females, Ratio of 2.1: 1). The maximum number of siblings per family was 8 and a minimum of 2 . The mean number of siblings per family was $4.75 \pm 1.77$.

The minimum duration from ingestion of foreign body to presentation to ER varied between 1 hour to 14 days with the median time of presentation to ER after ingestion of foreign body being 7 hours (Figure 1-8) Sixty five percent of the children were asymptomatic. There was abdominal pain in five (8\%) patients, most of them had ingested sharp objects which had passed beyond the duodenum and one patient had disc battery ingestion which was present in the stomach. Vomiting was the presenting symptom in seven patients (11\%), among them five patients had esophageal foreign bodies and the other two patients presented with foreign bodies in stomach and bowel. A history of choking was recorded in five (8\%) patients, all of these had ingested foreign bodies located in the esophagus. Increased salivation was present in one $(1.6 \%)$ patient with an esophageal foreign body. The other presenting symptoms that were recorded included, cough and dysphagia in one (1.6\%) patient with esophageal foreign body, blood with vomitus in one (1.6\%) patient (gastric foreign body). Nearly half (46\%) of patients with esophageal foreign bodies were symptomatic. 


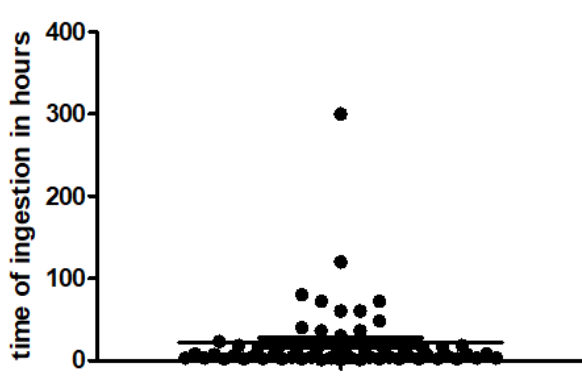

Figure 1: Time after ingestion.

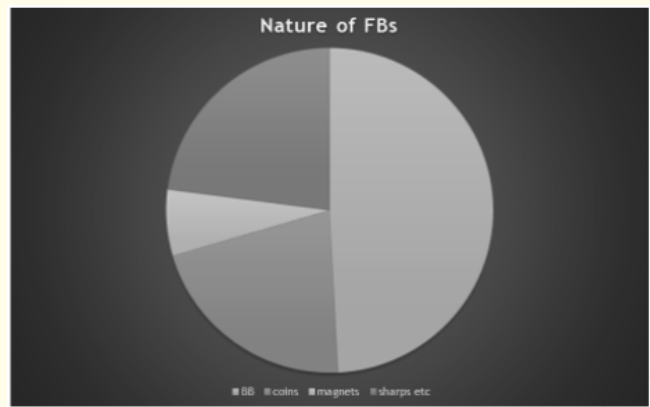

Figure 2: Nature of foreign bodies.

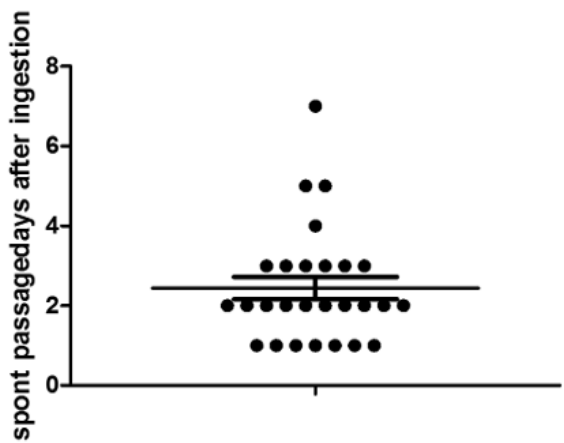

Figure 3: Spontaneous passage (days) after ingestion.

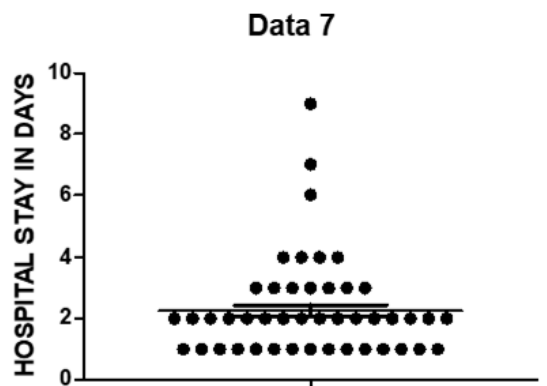

Figure 4: Average hospital stay (Days)
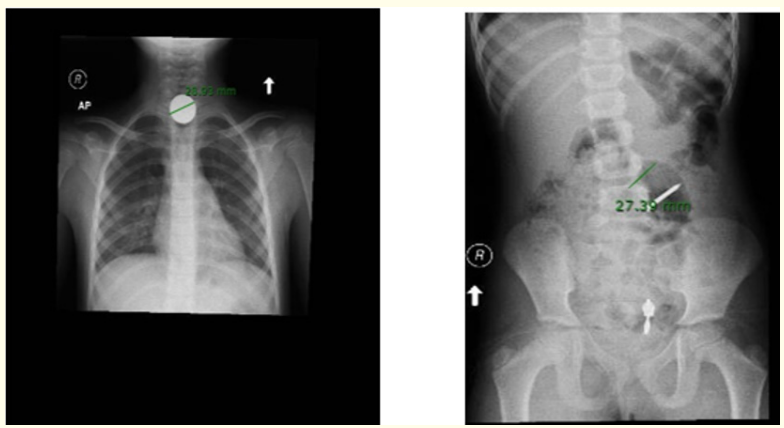

Figure 5: Showing an impacted coin and a sharp.
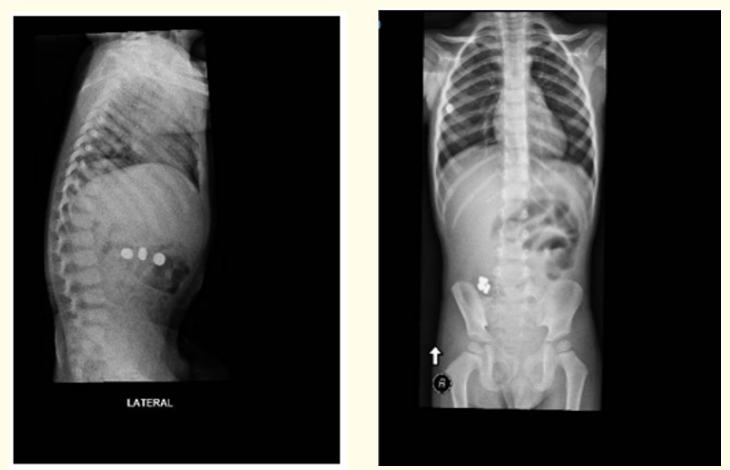

Figure 6: Showing multiple foreign bodies.
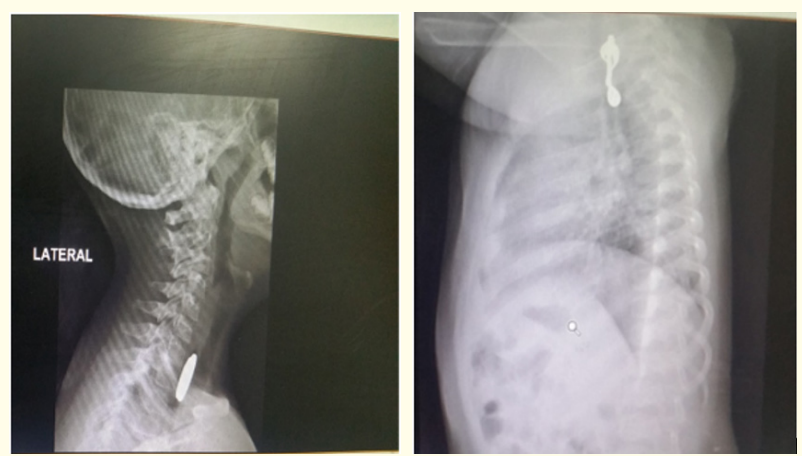

Figure 7: Step sign on lateral view (battery) and a complex foreign body (ear ring).

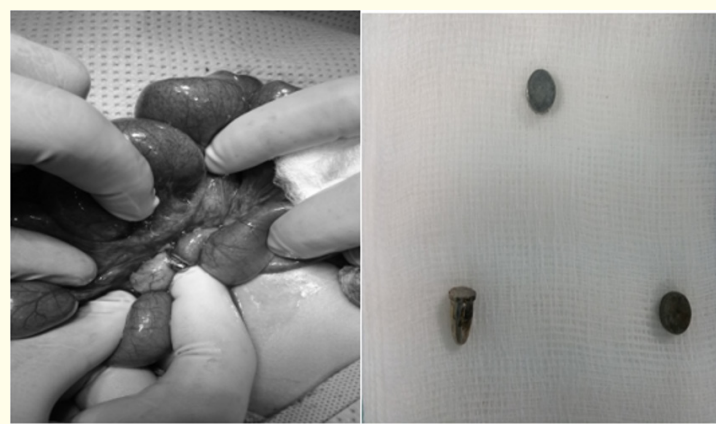

Figure 8: Magnet induced jejunal perforation. 
The ingested foreign bodies varied in sizes and diameters from less than a centimeter, $0.5 \mathrm{~cm}$ to $6.5 \mathrm{~cm}$ in case of sharps /blunt objects and the diameter ranged from $0.5 \mathrm{~cm}$ to $2.8 \mathrm{~cm}$ in case of ingested coins and button batteries The location of the foreign bodies on initial biplanar radiographs (anterioposterior and lateral) was as follows, Thorax and cervical region (esophagus): 24 (39\%) patients, Epigastrium and Left Upper Quadrant 19 (32\%) patients, Right lower quadrant 10 (16\%) patients , Left lower quadrant and pelvis $8(13 \%)$ patients.

Single foreign bodies were present in 56 (92\%) patients and the number was multiple in 5 (8\%) patients, with multiple magnet or battery ingestions. Recurrent ingestions over a period of 6 months was documented in one (1.6\%) patient.

The nature of the foreign bodies was as follows: Button batteries: 30 (49\%) patients, coins in $13(21 \%)$ patients, Magnets in 4 (7\%) patients. Sharp objects such as nails, screws pins and complex foreign bodies such as earrings, plastic pieces etc were present in $14(23 \%)$ patients.

Spontaneous passage of the foreign body over a period of observation was seen in 28 (46\%) patients. Laxatives (oral lactulose in a dose of $1 \mathrm{ml} / \mathrm{kg}$ ) were prescribed for 12 patients (20\%).

The mean number of days after which the ingested foreign body passed out spontaneously was $2.44 \pm 1.45$ days with a minimum duration being 1 day and maximum of 7 days.

Out of the total 61 patients, 38 (62\%) underwent interventions. Endoscopy was carried out for 37 (61\%) patients and surgery was performed for one $(1.6 \%)$ patient. Out of a total 37 patients who underwent endoscopy, thirty five patients had endoscopy for foreign body removal at our hospital, all of these were flexible endoscopies except one patient who required a rigid endoscopy. A total of $31(89 \%)$ patients had successful endoscopic removal of foreign bodies. In the remaining four (11\%) patients in whom endoscopy was performed at our hospital, the foreign bodies had already passed beyond the duodenum and were not amenable for endoscopic removal. There were two (3.3\%) patients who underwent failed esophagoscopy outside our hospital, a re- endoscopy was performed for 1 patient among these at our hospital but the foreign body had already passed beyond the duodenum. Laparotomy was indicated and performed for one $(1.6 \%)$ patient with a history of multiple magnet induced jejunal perforation. A total of five (14\%) patients out of the total 36 patients (including one patient who had a re-endoscopy at our hospital) had diagnostic endoscopy with no foreign body seen on endoscopy and all of these patients spontane- ously passed the foreign body over a period of time. The overall success for endoscopic foreign body removal was 86 percent $(31$ successful endoscopic removal of foreign bodies out of the total 36 endoscopies done at our center).

Timing of intervention, varied between 3 hours to 18 hours. Mean duration was $6.3 \pm 3.85$ hours Re-endoscopy was done in five (8\%) out of the total 61 patients. These patients had esophageal ulcers with granulation tissue on initial endoscopy, four patients among these had button battery ingestion with battery present in esophagus and one patient had impacted coin in esophagus. Gastric ulceration with granulation tissue was seen in $1(1.6 \%)$ patient with ingested battery in stomach. All these patients were given a course of proton pump inhibitor for 10 to14 days with an uneventful follow up.

The hospital stay of the patients varied between 1 to 9 days. The mean duration of hospital stay was $2.26 \pm 1.43$ days.

There were 21 (34\%) patients with available follow up records and all these patients had an uneventful course with no recorded re-admission or any secondary interventions.

\section{Discussion}

The advent of the modern era of electronics and gadgets has seen a dramatic increase in the number of ingested foreign bodies especially button batteries and magnets in children with subsequent need for medical care, advice and possible intervention. Most of the foreign bodies pass through the GI tract spontaneously without any significant morbidity and mortality. Our study aimed at reviewing the incidence and clinical profile of the children who were admitted at our hospital with history of ingested foreign bodies.

In this study 61 patients had their documented data recorded and analyzed. Most of the children in the study group were preschool age with a mean age of $5.13 \pm 2.68$ years (median of 4.9 years) which is slightly higher as reported by the American association of poison control centres 2000 [4]. Foreign body ingestions happened to be twice more frequent in boys as compared to girls and mostly in large families with the average number more than four children. The reason may possibly be lack of adequate care, close supervision and proper attention to every individual child by caregivers in large families.

Most of the children presented to the ER within the first 24 hours after the ingestion of foreign body with a median being 7 hours. The relatively higher mean time of ingestion as seen in (Fig- 
ure 1) was due to late presentation of one patient at 14 days after ingestion of foreign body. Inci etal and Kruk-Zagajewska., et al. $[20,21]$ in their studies reported that $90 \%$ of patients presented to ER within 10 hours of foreign body ingestion, where as Dereci., et al. [22] reported that $67 \%$ of patients reported to ER within 24 hours of ingestion.

Children may be completely asymptomatic or present with overt symptoms, including, but not limited to, stridor, drooling, irritability, chest pain, abdominal pain, fever, feeding refusal, wheezing, and respiratory distress [17]. In our study, $65 \%$ of the children were asymptomatic. Vomiting was the most common symptom present in $11 \%$ of patients followed by abdominal pain in $8 \%$ of the patients. Around $8 \%$ of the patients presented to ER with choking. The other presenting symptoms included increased salivation, cough with dysphagia and blood stained vomitus. Overall $46 \%$ of patients with esophageal foreign bodies were symptomatic.

Children tend to swallow foreign bodies of varying sizes and shapes. The ingested foreign bodies varied in sizes and diameters from less than a centimeter $(0.5 \mathrm{~cm})$ to $6.5 \mathrm{~cm}$ in case of sharps / blunt objects and the diameter ranged from $0.5 \mathrm{~cm}$ to $2.8 \mathrm{~cm}$ in case of ingested coins and button batteries.

Thorax and cervical (esophagus) was the most common site (39\%) of foreign body location on initial bi planar X-rays, followed by epigastric region and left upper quadrant (32\%), Right lower quadrant (16\%), left lower quadrant and pelvis (11\%). Little and Lin $[23,24]$ also reported esophagus to be the most common site of foreign body ingestion.

Although majority ( $>90 \%$ ) of foreign body ingestions are single, multiple ingested foreign bodies were seen in around $9 \%$ of patients with a small percentage of children having a recorded history of recurrent ingestions over the study period.

Button batteries were the most common foreign bodies ingested in our series with $39 \%$ cases, followed by coins $(21 \%)$, sharp objects, complex foreign bodies such as blunt objects, ear rings etc $(23 \%)$ and magnets (7\%). Of peculiar interest were complex foreign bodies like metallic ear rings, which were seen in $6 \%$ of patients. The incidence of button battery ingestion is relatively higher in the study group which is a worrisome trend. Most of the literature reports coins to be the most common form of ingestion. Coins are the most common ingested objects among children in the United States [25]. In a study from Belgium by Arana., et al. (2001) with 325 children, the rates of ingestion of coins were $27 \%$, followed by toy parts, jewels and batteries in decreasing order [26]. The high incidence of button battery ingestion, as was noticed in our results, may be due to increased access to electronic toys and gadgetry especially with introduction of cheap and poor quality materials with no proper safety locks for securing the batteries due to poor safety and manufacturing protocols followed by these manufacturers. Furthermore improper disposal of used batteries by caretakers may be compounding the problem as well.

Around $70 \%$ to $80 \%$ of the foreign bodies do not need any intervention and pass spontaneously without any significant morbidity and mortality $[2,5]$. In around half of the patients $(46 \%)$, the foreign body passed spontaneously over a period of observation with a mean number of 2.44 days (range being 1 day to 7 days). Although the percentage of patients with spontaneous passage of foreign bodies is less in our series, compared to that reported by Webb and Carp [2,5], it is due to the fact that incidence of button battery was much higher in our series (39\%) and the most common site involved was the oesophagus, which made emergency removal mandatory.

In our study out of a total of 61 patients, 38 (62\%) underwent interventions, endoscopy or surgery. One patient needed a laparotomy for multiple magnet ingestion induced jejunal perforation. The rate of use of endoscopy to remove foreign bodies has been reported to be $34 \%$ by Aydoglu., et al. [27] and 25\% by Arana., et al. [26] .The high incidence of interventions in our series may be related to a relatively higher incidence of button battery ingestions with a significant number of foreign bodies, batteries, coins and complex objects, impacted in esophagus and stomach.

There were 7 patients (12\%) who had complications, jejunal perforation in one patient and six patients had esophageal/gastric ulcers on initial endoscopy. Five patients among these required a follow up re endoscopy.

There were two patients who presented to our hospital outside our study period, one with button battery induced esophageal perforation with mediastinitis and pyothorax and the other child presented with a battery induced esophageal stricture with failed medical management. Both of these required thoracotomy with a prolonged ICU and hospital stay with a long time for recovery.

\section{Conclusion}

Foreign body ingestion in children is a serious problem of large magnitude. The high number of hospital admissions to one single centre in the region is really a cause of concern. Moreover the number of button batteries ingestions recorded is alarmingly high, which can be potentially life threatening, and puts extra burden on the health care workers with increased need for interventions. 
There is an urgent need for an awareness campaign to be launched by the authorities to educate parents about the hazards of foreign body ingestionsespecially button batteries. Moreover the battery operated toys and appliances available in the market should be checked for quality and proper manufacturing safety protocols.

\section{Conflicts of Interest}

There were no conflicts of interest.

\section{Acknowledgement}

This study was not possible without the suppost of the KSMC research center and and the medical records department.

\section{Bibliography}

1. Wyllie R. "Foreign bodies in the gastrointestinal tract". Current Opinion in Pediatrics 18 (2006): 563.

2. Webb WA. "Management of foreign bodies of the upper gastrointestinal tract: update". Gastrointestinal Endoscopy 41.1 (1995): 39-51.

3. Waltzman ML., et al. "A randomized clinical trial of the management of esophageal coins in hildren". Pediatrics 116.3 (2005): 614-619.

4. Litovitz TL., et al. "2000 annual report of the American Association of Poison Control Centers Toxic Exposure Surveillance System". American Journal of Emergency Medicine 19.5 (2001): 337-395.

5. Carp L. "Foreign bodies in the intestine". Annals of Surgery 85.4 (1927): 575-591.

6. Uyemura MC. "Foreign body ingestion in children". American Family Physician 72.2 (2005): 287-291.

7. Shivakumar AM., et al. "Foreign body in upper digestive tract". Indian Journal of Pediatrics 71.8 (2004): 689-693.

8. Palta R., et al. "Foreign-body ingestion: characteristics and outcomes in a lower socioeconomic population with predominantly intentional ingestion". Gastrointestinal Endoscopy 69.31 (2009): 426-433.

9. Weiland ST and Schurr MJ. "Conservative management of ingested foreign bodies". Journal of Gastrointestinal Surgery 6.3 (2002): 496-500.

10. Athanassiadi K., et al. "Management of esophageal foreign bodies: a retrospective review of 400 cases". European Journal of Cardio-Thoracic Surgery 21.4 (2002): 653-656.
11. Reilly $S$ and Carr L. "Foreign body ingestion in children with severe developmental disabilities: a case study". Dysphagia 16.1 (2001): 68-73.

12. Louie JP., et al. "Witnessed and unwitnessed oesophageal foreign bodies in children". Pediatric Emergency Care 21.9 (2005): 582-585.

13. Yalcin S., et al. "Foreign body ingestion in children: an analysis of pediatric surgical practice". Pediatric Surgery International 23.8 (2007): 755-761.

14. Conners G P. “Pediatric Foreign Body Ingestion”. (2015).

15. Antonacci N., et al. "A bizarre foreign body in the appendix: a case report". World Journal of Gastrointestinal Surgery 5.6 (2013) 195-198.

16. Susan E. Foreign bodies in the esophagus in Nelson Textbook of Pediatrics, R. E. Behrman, R. M. Kligman, and H. B. Jenson, Eds., p. 1552, Saunders, Philadelphia, Pa, USA, 17th edition (2007).

17. Chen MK and Beierle EA. "Gastrointestinal foreign bodies". Pediatric Annals 30 (2001):736-742.

18. Dahshan A. "Management of ingested foreign bodies in children". Journal of the Oklahoma State Medical Association 94 (2001): 183-186.

19. Eisen GM., et al. "Guideline for the management of ingested foreign bodies". Gastrointestinal Endoscopy 55.7 (2002): 802806.

20. İnci İ., et al. "Özofagus Yabancı Cisimleri: 682 olgunun incelenmesi". GKDC Dergisi 7 (1999): 148-152.

21. Kruk-Zagajewska A., et al. "Foreign bodies in the esophagus". Polish Journal of Otolaryngology 53 (1999): 283-288.

22. Dereci S., et al. "Foreign body ingestion in children". Turkish Archives of Pediatrics/Türk Pediatri Arşivi 50.4 (2015): 234-240.

23. Little DC., et al. "Esophageal foreign bodies in the pediatric population: our first 500 cases". Journal of Pediatric Surgery 41.5 (2006): 914-918.

24. Lin HH., et al. "Emergency endoscopic management of dietary foreign bodies in the esophagus". American Journal of Emergency Medicine 25.6 (2007): 662-665.

25. Chen X., et al. "Pediatric coin ingestion and aspiration". International Journal of Pediatric Otorhinolaryngology 70.2 (2006): 325-329. 
Foreign Body Ingestion in Children: A Hospital Based Experience in Riyadh

26. Arana A., et al. "Management of ingested foreign bodies in childhood and review of the literature". European Journal of Pediatrics 160.8 (2001): 468-472.

27. Aydogdu S., et al. "Foreign body ingestion in Turkish children". The Turkish Journal of Pediatrics 51 (2009): 127-132.

Volume 2 Issue 10 October 2019

(c) All rights are reserved by Zubair Khurshid., et al. 